\title{
RAG knockouts deliver a one/two punch
}

\author{
Mice lacking recombinase activating gene 1 or 2 are devoid \\ of mature B and T cells but are otherwise developmentally \\ normal. They may be ideal for transplantation studies.
}

The ability of mammals to protect themselves from invasion by foreign cells or pathogens is controlled primarily by the lymphoid immune system. This system is composed of $\mathrm{B}$ cells, which produce antibodies, and $\mathrm{T}$ cells, which direct cell-mediated immune responses. Collectively, mature $B$ and $T$ cells have the ability to initiate immune responses against an enormous number of foreign antigens. Recognition of antigens by $B$ cells is mediated through the variable region of their cell surface immunoglobulin receptor (sIg). T-cell antigen recognition is mediated by the variable domain of the T-cell receptor (TCR). The genes that encode the sIg and TCR molecules are assembled during $\mathrm{B}$ and $\mathrm{T}$ cell development, respectively, from individual members of variable (V), diversity (D), and joining (J) gene segment families in a process known as V(D)J recombination. Although $V(D) J$ recombination creates only a single type of TCR or sIg in each individual $\mathrm{B}$ or $\mathrm{T}$ cell, the combinatorial diversity of the recombination process generates $B$ and $T$ cells with $>10^{8}$ distinct antigenic specificities. Subsequent selection processes during B-and T-cell development serve to remove or inactivate cells with receptors that react against self-antigens.

Although many of the cis-acting elements that regulate the V(D)I recombination process have been defined, relatively little is known about the V(D)J recombinase itself. Present evidence suggests that the same recombinase mediates the rearrangement of both immunoglobulin and T-cell receptor genes. As currently conceived, the V(D)J recombinase must be able to carry out a series of complex reactions, including: recognition of the recombination signal sequences that flank $\mathrm{V}, \mathrm{D}$, and $J$ elements; precise cleavage of the DNA substrate at the border between the signal and coding sequences; pairing and ligation of two signal sequences; modification of the end of the coding sequence, including basepair addition and deletion; and coding sequence endligation (Fig. 1). Based on the large number of events that occur during recombination, it has long been assumed that recombinase activity might require the concerted function of several gene products. Some of the recombinase components are likely to be lymphoid-specific, whereas others may play a more general role in DNA repair. Consistent with this hypothesis, the murine scid mutation, which involves a generalized deficiency in double strand DNA break repair, profoundly inhibits the coding sequence ligation step of V(D)J recombination [1]. As a result the ability of scid mice to develop functional $B$ and $T$ cells is impaired and they develop a severe combined immunodeficiency.
In 1990, two murine genes, recombinase activating gene (RAG) 1 and 2, were isolated by virtue of their ability to synergistically activate $\mathrm{V}(\mathrm{D}) \mathrm{J}$ recombination in fibroblasts [2]. The two genes are unrelated in amino acid sequence but are located only 8 kilobases $(\mathrm{kb})$ apart on the same chromosome. Although the $5^{\prime}$-untranslated regions of the cDNAs of both genes appear to contain spliced exons, the coding sequences of both RAG1 and RAG-2 are contained within a single exon. The two genes and their organization have been highly conserved in vertebrate evolution. Coexpression of RAG-1 and RAG-2 has been observed only in developing lymphoid cells and lymphoid cell lines that have V(D)J recombinase activity. Based on these results, it appears that RAG-1 and RAG-2 together either activate the expression of the $\mathrm{V}(\mathrm{D}) \mathrm{J}$ recombinase or are themselves both essential components of the recombinase. RAG-1 has been reported to have limited sequence similarity to the yeast gene Hpr-1, which plays a role in regulation of recombination events in yeast, but RAG-2 has not been found to be related to any known gene product. To date, the properties of the protein products of RAG-1 and RAG-2 have not been characterized. Thus, the individual roles of these two genes in the regulation of $V(D) J$ recombination has not been established.

Recombination as a genetic control mechanism has been observed frequently in prokaryotes and single cell eukaryotes. With the exception of immunoglobulin- and TCRassociated recombination events, however, developmental recombination has not been observed in vertebrates, despite numerous suggestions that recombination may have additional roles in vertebrate embryogenesis and neural development. It has been speculated that expression of either RAG-1 or RAG-2 alone might have roles in developmental recombination events that are distinct from V(D)J joining. Consistent with this hypothesis, isolated expression of RAG-1 has been reported in the murine central nervous system and isolated expression of RAG-2 has been observed in the chicken bursa of Fabricius $[3,4]$. The possibility that RAG-1 might play a part in the regulation of genetic recombination in the brain was increased by recent evidence [5] of the rearrangement of a transgene containing $V(\mathrm{D}) \mathrm{J}$ recombination signal sequences within the central nervous system as well as within lymphoid cells - a pattern that mimics the expression of RAG-1.

To test the role of RAG-1 and RAG-2 in murine development, separate groups have now performed targeted disruption of each of the genes in the mouse germline and characterized the developmental effects of 

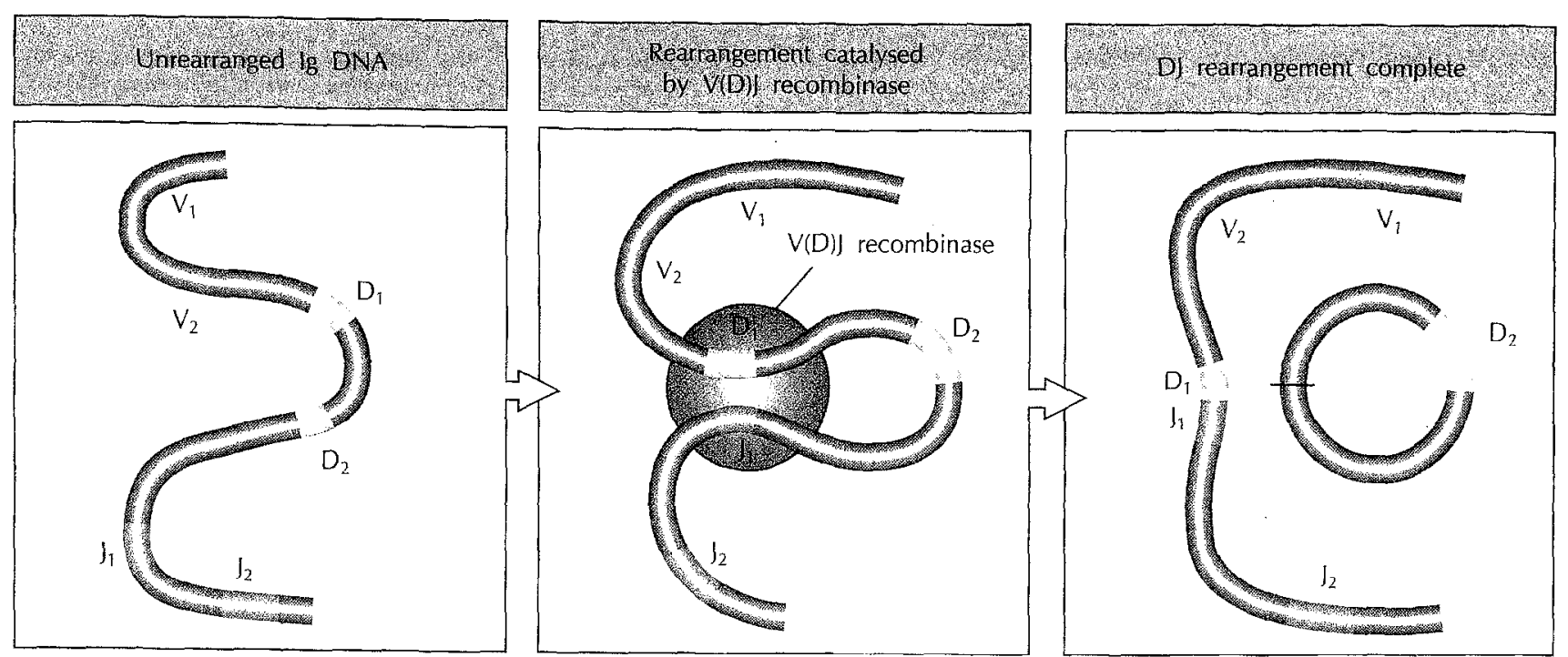

Fig. 1. Schematic represeritation of DJ rearrangement catalysed by the V(D)] recombinase. Once a DJ recombination has occurred, the rearranged DJ segment bccomes a substrate for a second recombination in which the $V$ segrnent is joined to the rearranged DJ segment. This second rearrangement (not shown) is also directed by recombination signal sequences (red) and catalysed by the V(D)f recombinase.

homozygous deletion of that gene. Mombaerts et al. [6] disrupted RAG-1 in the mouse germline, while Shinkai et al. [7] disrupted RAG-2. Interestingly, both RAG-1 and RAG-2 deficient mice appear to have identical phenotypes - their lymphoid organs are devoid of mature $B$ and $\mathrm{T}$ cells. The arrest of $\mathrm{B}$ - and T-cell development occurs at the earliest known stages, preventing a significant accumulation of immanure lymphoid cells. No observable effects on other haematopoietic cell lineages, including natural killer cells and myeloid cells, were noted.

In both sets of mice, it was possible to show that B-lymphoid development had beelı initiated; pro-B cells were detected by means of their immortalization with Abelson murine leukemia virus ( $\Lambda$-MuLV). Immortalized cells from both sets of mice expressed a variety of early B-lineage markers including the B-lineage isoform of CD45, $\lambda 5$, and the non-deleted RAG product. Immunoglobulin genes were present only in germline configuration. In immortalized pro-B cells from RAG-2 deficient mice, the $\mathrm{V}(\mathrm{D}) \mathrm{J}$ recombination defect could be corrected by transfection of a functional RAG-2 gene. (A similar experiment with RAG-1 cells has not yet been performed.) These data suggest that RAG-deficient $B$ lymphocytes are arrested at the point where they might be expected to initiate $\mathrm{D}$ to $\mathrm{J}$ recombination.

T-cell development in RAG-deficient mice also appears to be arrested at a very early stage. Within the thymus, cells coexpressing the Thy- 1 antigen and the $\alpha$ chain of the interleukin-2 receptor can be detected. This is the phenotype of the earliest $T$ cells that colonize the normal thymus. However, the cells have no detectable TCR gene rearrangements and do not express CD4 and $\mathrm{CD} 8$, even though normal thymocytes express these coreceptor molecules before expressing T-cell receptors on their surface. This suggests, somewhat surprisingly, that RAG gene function is necessary before cells acquire CD4 and $\mathrm{CD} 8$ expression. The data suggest that $\mathrm{T}$ cells have an intermediate stage of development during which they undergo only $\mathrm{D}$ to J rearrangement. Apparently T cells cannot proceed past this point in development in the absence of RAG-1 and RAG-2 co-expression.

Apart from the complete deficiency of $\mathrm{B}$ and $\mathrm{T}$ cells, neither RAG-1 nor RAG-2 deficient mice had any observable developmental defects. Mating either RAG-1 or RAG-2 heterozygotes produced homozygotic mutant mice at a frequency of approximately $25 \%$. Thus, disruption of neither gene resulted in a significant rate of embryonic lethality. Both RAG-1 and RAG-2 deficient mice were fertile. Both sets of mice have been examined in considerable detail for non-lymphoid pathology, but there were no observable macroscopic, histochemical, or behavioural differences between RAG-deficient mice and control litter mates. Together, these results suggest that neither the recombinase activating genes or $\mathrm{V}(\mathrm{D}) \mathrm{J}$ recombinase activity is absolutely required for the development of non-lymphoid cells.

No defects in the development of the central nervous system of RAG-1 deficient mice were seen. The animals have been observed for up to 21 weeks and have apparently failed to develop any neurological abnormalities. The animals can reportedly see, hear, and feel normally, have good motor strength and coordination, and can carry out patterned behavior. The gross and histologic anatomy of the brain suggested that the disruption of RAG-1 function does not interfere with neural development. Thus, previously reported [5] central nervous system recombination events are either not required for neural development or do not involve RAG 1 . The present data, however, do not completely rule out a role for RAG-1 in the maintenance of the central nervous system. It has been speculated that RAG-1 may play a role in the suppression of illegitimate recombination in long lived cells such as neurons. Long term observation of the animals and repeated histological examinations will be needed to address this hypothesis. 
As RAG-1 and RAG-2 deficient animals do not appear to have developmental abnormalities outside the lymphoid immune system, the results of Mombaerts et al. and Shinkai et al. have important implications for investigators who study hematopoietic development. One of the major barriers to the study of human hematopoietic cells in animal models has been the transplantation barrier created by the lymphoid immune system. Recently, several important human hematological model systems have been established using human bone marrow transplanted into immunodeficient mice [8,9]. For example, scid mice reconstituted with human bone marrow have been used to study human immunodeficiency virus infection in vivo as well as to define the in vivo effects of human growth factors and chemotherapeutic agents. However, the use of scid mice has two potential drawbacks: scid mice have a generalized defect in DNA repair, and the scid immune defect is leaky. As scid animals age, mature $B$ and T cells begin to appcar and thesc cclls may mediate transplantation rejection in some animals.

As already noted, mice deficient in RAG-1 or RAG-2 have a phenotype very similar to that described for scid mice. In contrast to scid mice, however, no leakiness has been observed in the block to lymphocyte development in RAGdeficient mice. This suggests that RAG-deficient mice may have real advantages for the tissue transplantation studies currently being undertaken in either scid or nude mice. For example, RAG-deficient mice will be ideal for the production of high titer antibodies from myeloma cells because they are completely deficient in endogenous antibody production. RAG-deficient mice should also be useful for testing and studying the growth of allogeneic and xenogeneic tumours, and they may be even more effective than scid mice at supporting long-term growth of human hematopoietic cells. If so, RAG-deficient mice may be used to facilitate the development of murine models of human hematopoietic diseases.

\section{References}

1. BOSMA MJ, CARROLL AM: The scid mouse mutant: definition, characterization and potential uses. Annu Rev Immunol 1991 $9: 323-350$.

2. Oettinger MA, Schatz DG, Gorka C, Baltimore D: RAG-1 and $R A G-2$, adjacent genes that syncrgistically activate $V(D) J$ recombination. Science 1990, 248:1517-1522.

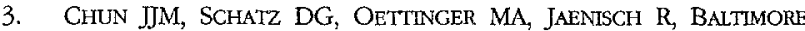
$D$ : The recombination activating gene-1 (RAG-1) transcript is present in the murine central nervous system. Cell 1991 64:189-200.

4. Carlson LM, OETTINGER MA, Schatz DG, Masteller EL, HURLey EA, MCCORMACK WT, BALTIMORE D, THOMPSON CB; Selective expression of RAG-2 in chicken $B$ cells undergoing immunoglobulin gene conversion. Cell 1991, 64:201-208.

5. MATSUOKA M, NAGAWA F, OKAZAKI K, KINGSBURY L, YOSHIDA K, MULLER U, LARUE DT, WINER JA, SAKANO H: Detection of somatic DNA recombination in the transgenic mouse brain. Science 1991, 254:81-86.

6. MOMBaERTS $P$, IACOMINI $J$, JOHNSON RS, Herrup $K$, TONEGAWA $\mathrm{S}$, PAPAIOANNOU VE: RAG-2 deficient mice have no mature $B$ and T lymphocytes. Cell 1992, 68:869-877.

7. SHINkaI $Y$, Rathbun G, LAM K-P, Oltz EM, Stewart V, MENDEISOHN M CHARON J, DATTA M, YOUNG F, STALl AM, ALT FW: RAG-2 deficient mice lack mature lymphocytes due to inability to initiate VDJ rearrangement. Cell 1992, 68:855-867.

8. MCCUNE JM, NAMIKAWA R, KANESHIMA $H$, SCHULTZ LD, LIEBERMAN M, WEISSMAN IL: The SCID-hu mouse: murine model for the analysis of human hematolymphoid differentiation and func tion. Science 1988, 241:1632-1639.

9. KAMEL-REID S, DICK JE: Ingraftment of immunc-deficient mice with human hematopoietic stem cells. Science 1988 , 242:1706-1709.

Craig B. Thompson, Howard Hughes Medical Insti tute, Department of Internal Medicine and Microbiology/Immunology, University of Michigan Mcdical Center, Ann Arbor, Michigan 48109, USA.

\section{THE APRIL 1992 ISSUE OF CURRENT OPINION IN IMMUNOLOGY}

Contains the following reviews on Lymphocyte Development, edited by Klaus Rajewsky and Harald von Boehmer:

Early B-cell development in chicken, sheep and rabbit by $\mathrm{J}$ Weill and $\mathrm{C}$ Reynaud Distinctions in V gene usage, physiology and development of $\mathrm{Ly}-\mathrm{I}\left(\mathrm{CD}^{+}\right)$B cells by R Hardy Development and selection of $\gamma \delta$ T-cells by W Häas and S Tonegawa The generation of the $\alpha: \beta T$ cell repertoire by $C$ Benoist and D Mathis Extrathymic T-cell selection by B Arnold, G Schönrich and G Hämmerling Cellular aspects of early $\mathrm{T}$-cell development by $\mathrm{K}$ Shortman

Thymic selection by D Pardoll and A Carrera

Late B-cell development by $\mathrm{K}$ Rajewsky Hematopoietic stem cells by $G$ Keller 